\title{
Design of Laboratory Experiments to study Radiation-Driven Implosions
}

\author{
P. A. Keiter, M. Trantham, G. Malamud, S. R. Klein, J. Davis, R. VanDervort, D. Shvarts, R. P. Drake \\ University of Michigan, Ann Arbor, MI 48105
}

J. M. Stone

Princetone University, Princeton, NJ 08544

M. Fraenkel, Y. Frank, E. Raicher

Soreq Research Center, Israel

\begin{abstract}
The interstellar medium is heterogeneous with dense clouds amid an ambient medium. Radiation from young OB stars asymmetrically irradiate the dense clouds. Bertoldi (1989) developed analytic formulae to describe possible outcomes of these clouds when irradiated by hot, young stars. One of the critical parameters that determines the cloud's fate is the number of photon mean free paths in the cloud. For the extreme cases where the cloud size is either much greater than or much less than one mean free path, the radiation transport should be well understood. However, as one transitions between these limits, the radiation transport is much more complex and is a challenge to solve with many of the current radiation transport models implemented in codes. We present the design of laboratory experiments that use a thermal source of x-rays to asymmetrically irradiate a low-density plastic foam sphere. The experiment will vary the density and hence the number of mean free paths of the sphere to study the radiation transport in different regimes. We have developed dimensionless parameters to relate the laboratory experiment to the astrophysical system and we show that we can perform the experiment in the same transport regime.
\end{abstract}

\section{Introduction}

Ionization fronts, pillars and star formation are just some of the well-known phenomena produced when radiation from hot young OB stars interacts with its surrounding medium. The interstellar medium is heterogeneous, with dense regions, often referred to as clouds or clumps within a less dense medium. The photons emitted by stars interact with a heterogeneous interstellar medium. Figure 1 shows a basic representation. An important aspect of this problem is that the radiation is incident on only one side of the cloud. The optical depth of the cloud to the incident radiation is a major factor in determining the fate of the cloud. For example, optically thin clouds may be heated and expand while optically thick clouds may be compressed.

Oort and Spitzer [1] were among the first to study the interaction of photons from young OB stars. They assumed the clouds are spherical and optically thick

Email address: pkeiter@umich.edu (P. A. Keiter)

Preprint submitted to Elsevier

\section{Photons}

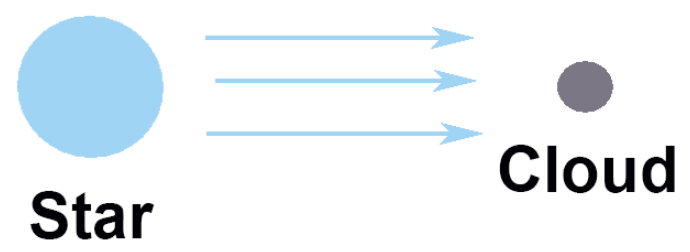

Figure 1: For this class of problem, generically one has a star, which emits radiation that is incident on one side a cloud.

to the UV radiation of the stars. The radiation is absorbed in a thin layer at the surface of the cloud, which heats the material and drives a shock into the cloud. Ionized gas is ablated from the cloud, and due to the 'rocket effect' the cloud is accelerated away from the star. However, in considering this phenomena, they ig-

October 31,2016 
nored the hydrodynamic evolution of the cloud itself, assuming a constant size during the interaction. Kahn [2] considered the problem in more detail and presented on the cloud velocity and mass in regions where early stars were present. However, he still did not consider the hydrodynamic evolution of the cloud. Klein et al. [3] and Sandford et al. [4] performed the first timeresolved, axisymmetric radiation hydrodynamic simulations of this problem. They considered radiation from an OB star incident on a dense (optically thick) cloud. Their work followed the compression, also referred to as the radiation-driven implosion (RDI), of the cloud. They showed that the cloud geometry can affect the details of the evolution. Bertoldi [5] considered the problem more generally, developing an analytic formula to describe the fate of the cloud based on the column density of the cloud and the incident flux from the star. He considered radiation fluxes and densities that varied over orders of magnitude, which covered clouds being heated and evaporated to the radiation-driven implosion. While the primary focus in the literature is on optically thick clouds because radiation-driven implosions are one mechanism for star formation, the case of the cloud diameter being $\sim 1$ optical depth is a challenge for radiation-hydrodynamic codes to solve.

Work by Kessel-Deynet and Burket [6] explored the aspect of star formation via the radiation driven implosion. However, there were a number of questions that were still outstanding, including: Where in the dense cloud does the star formation occur? When in the compression phase does it occur? What is the mass in the region? How are binary stars formed via this mechanism?

Bisbas et al. [7] performed a series of simulations varying the incident flux of photons from a star to attempt to answer many of these questions. They developed an analytic formula for the time that one would expect a star to form. They also note that a lower incident photon flux results in more massive stars, while a higher photon flux leads to less massive stars. In both cases, the shock sweeps up materials, compressing it highly. In the high flux limit, the shock is faster, compressing the material more quickly. The swept-up layer becomes unstable and fragments before it can laterally contract under its own self-gravity. Bisbas et al. [7] also considered the effect numerical noise had on their simulations and found that it did influence the results of when and where the star formation occurs and hence what the final mass is.

Recently, Proga et al. [8] performed two-dimensional time-dependent radiation hydrodynamics of strongly irradiated clouds, focusing on the role of opacity on the cloud evolution. In the case where the cloud opacity is dominated by absorption, the cloud does not move as a whole. The cloud evolves in shape and size and becomes optically thin in less than one sound-crossing time. When scattering dominates the opacity, the cloud can be accelerated as a whole. The acceleration due to the radiation force is small and the cloud morphology changes quickly unless the cloud is optically thin.

We present an experimental platform to study the radiation-driven implosion of a cloud. The purpose of the platform is to study the radiation transport as the cloud transitions from an optically thin to an optically thick regime. We discuss the scaling of the dimensionless variables introduced by Bertoldi [5] and derive the bounds achievable in the laboratory. While the initial experiments for this platform will utilize an optically thick cloud, the platform allows one to vary the optical thickness to study regimes of radiation transport that will challenge current radiation hydrodynamic codes.

\section{Experimental Design}

We have designed an experimental platform, which explores the radiation-hydrodynamic evolution of "clouds" with optical depths ranging from optically thin to optically thick to the incident radiation. Our experiments do not include gravity, but this is not required to study the radiation hydrodynamic effects. Although a star drives a photoionization front that is incident on a cloud, we will not require a photoionization front for the experiment as the requirements of creating a photoionization front in the laboratory are quite extensive. [9] The experiments have been initially designed with 1D codes HYADES [10] and HELIOS-CR [11] and the CRASH code [12] used in 2D. Following the simple representation of the problem in Figure 1, we need two key components: a radiation source and a cloud (Figure 2).

A key aspect of the experiment is to span the different regimes discussed by Bertoldi [5]. Figure 3 is adapted from Figure 1 in Bertoldi [5] showing the parameters one would expect the RDI mechanism to create a star.

There are two regions where one would not expect the RDI to compress the cloud to the required density for star formation. The blue region represents the situation where the radiation flux from the star is too low to produce a significant amount of pressure on the cloud and it weakly ablates material from the cloud. The gold region represents the situation where the radiation flux is high and the cloud is a small number of radiation mean free paths across. In the case there the cloud diameter is $\sim 1$ mean free path, complex behavior occurs. Bertoldi [5] 


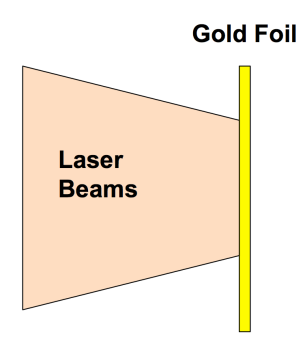

Figure 2: Laser beams are focused to a finite spot size and irradiate a thin gold foil, producing radiation which is asymmetrically incident a foam sphere. The density of the foam sphere can be varied to change the number of photon mean free paths across the sphere is and hence change the regime of radiation transport investigated.

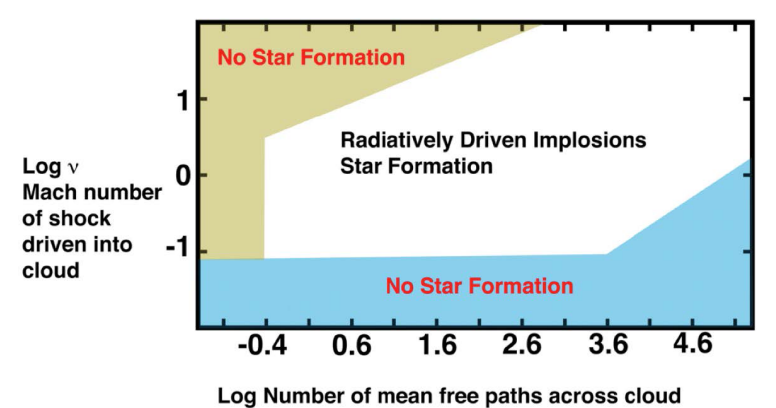

Figure 3: This figure has been adaptded from Figure 1 in Bertoldi [5] and illustrates the parameters at which one would expect the radiationdriven implosion mechanism to lead to star formation. terms this region as a nonequilibrium ionization front and indicates that a steady state ionization front can not be established until the shock driven by the incident radiation. The cloud evolution is therefore intrinsically time-dependent and is a challenge for current radiation hydrodynamic codes to model. As the radiation flux continues to increase, the cloud is heated and expands into the surrounding medium.

In the follow subsection, we discuss appropriate dimensionless parameters to relate the experiment to the astrophysical system and what parameters we can reasonably achieve in the laboratory.

\subsection{Consideration and scaling}

As discussed previously, Bertoldi [5] evaluates a cloud's fate from two parameters, $\eta$ and $v$, where $\eta$, is the maximum column density in the initial cloud and $v$ is the ratio of the initial shock velocity to the sound speed of the ambient medium. Bertoldi finds the initial shock velocity by equating the shock ram pressure to the total pressure at the D-critical ionization front where $n_{0} c_{i}{ }^{2}=2 F_{i} c_{i}$. We now consider each of these parameters and discuss the ranges one can achieve in laboratory experiments. First, we consider $\eta$, which Bertoldi [5] defined as:

$\eta=\frac{\alpha_{i} r_{0} n_{0}}{c_{i}}=\frac{r_{0} n_{0}}{4.4 \times 10^{18} \mathrm{~cm}^{-2}}$

where $\alpha$ is the rate coefficient for hydrogen recombinations to all levels but the ground states, $r_{0}$ is the initial radius of the cloud, $n_{0}$ is the initial density of the cloud and $c_{i}$ is the isothermal sound speed of the intercloud gas. The final form is found by evaluating this for typical interstellar parameters.

When dealing with radiation, it is often useful to consider the number of mean free paths in the system, where $\lambda_{0}=\frac{1}{n_{o} \sigma_{H}}$, is the mean free path. We can relate the number of mean free paths across an object, $\frac{r_{0}}{\lambda_{0}}$ to $\eta$. $\frac{r_{0}}{\lambda_{0}}=r_{0} n_{0} \sigma_{H}$. The cross section of hydrogen is on order $1 \mathrm{Mb}=10^{-18} \mathrm{~cm}^{-2}$, therefore $\frac{r_{0}}{\lambda_{0}}=4.4 \eta$.

Now we consider $v$, which is defined as

$v=\left(\frac{2 F_{i}}{n_{0} c_{i}}\right)^{1 / 2}$

where $c_{i} \sim \sqrt{\frac{Z T_{s}}{M}}$. Therefore $v=\left(\frac{2 F_{i}}{n_{0}} \sqrt{\frac{M}{Z T_{s}}}\right)^{1 / 2}$.

We evaluate these dimensionless variables to find the phase space we can explore in the laboratory. First 
we consider the radiation source to drive the experiment. Ideally the source will provide a constant radiation flux over the lifetime of the experiment. Hohlraums have been used as radiation sources in many radiation transport experiments because they can reach high (> $200 \mathrm{eV}$ ) radiation temperatures and have durations of many ns. However, the radiation temperature of the hohlraum varies in time, hohlraums produce $\sim 10-15$ $\%$ M-band radiation, which can preheat the target components and the stagnation of the laser-irradiated gold on axis can produce an axial jet that can then interact with the physics package. Recent experiments irradiating thin gold foils with lasers demonstrated $\mathrm{a} \sim 6 \mathrm{~ns}$ radiation source with an effective temperature of $\sim 100$ $\mathrm{eV}$. [13] To access a regime where the sphere diameter is roughly 1 photon mean free path, one can lower the density of the sphere by considering plastic foams, which have densities down to $\sim 1 \mathrm{mg} / \mathrm{cm}^{3}$. We evaluate the ionization, $\mathrm{Z}$, for a $\mathrm{CH}$ plastic sphere (atomic weight of 6.5) irradiated at $100 \mathrm{eV}$, to be $\mathrm{Z} \sim 2.5$. For a $\mathrm{CH}$ foam with a density of $10 \mathrm{mg} / \mathrm{cm}^{3}$ and a photon flux of $10^{29}$ photons $/ \mathrm{cm} / \mathrm{s}$, we find a value of 8.5 for $v$. We can reduce $v$ by lowering $\mathrm{F}_{i}$ and can reasonably obtain values of $v$ in the range of 0.1 to 10 . We summarize the ranges for a number of important parameters one finds in both the astrophysical and laboratory systems in Table 1 .

Table 1: Scaling parameters

\begin{tabular}{|c|c|c|}
\hline Parameter & $\begin{array}{c}\text { Astrophysical } \\
\text { RDI }\end{array}$ & $\begin{array}{c}\text { Laboratory } \\
\text { RDI }\end{array}$ \\
\hline $\mathrm{C}_{s}(\mathrm{~cm} / \mathrm{s})$ & $1.14 \times 10^{7}$ & $10^{7}$ \\
\hline $\mathrm{N}\left(\mathrm{cm}^{-3}\right)$ & 500 & $10^{19}-10^{21}$ \\
\hline $\mathrm{F}$ (photons $\mathrm{s}^{-1} \mathrm{~cm}^{-2}$ ) & $10^{8}-10^{9}$ & $10^{27}-10^{29}$ \\
\hline$v$ & $0.1-10$ & $0.1-10$ \\
\hline$\eta$ & $0.1-10^{4}$ & $0.1-10^{5}$ \\
\hline Optical Depths & $10-50$ & $\leq 50$ \\
\hline
\end{tabular}

The first experiments to develop this platform will concentrate on optically thick clouds. We will use xray radiography from one or more directions to diagnose the temporal evolution of the shock driven into the foam sphere.

\section{Simulation results}

We performed initial simulations for the platform with the CRASH radiation-hydrodynamics code [12]. CRASH includes a laser package and uses multigroupdiffusion radiation transport.

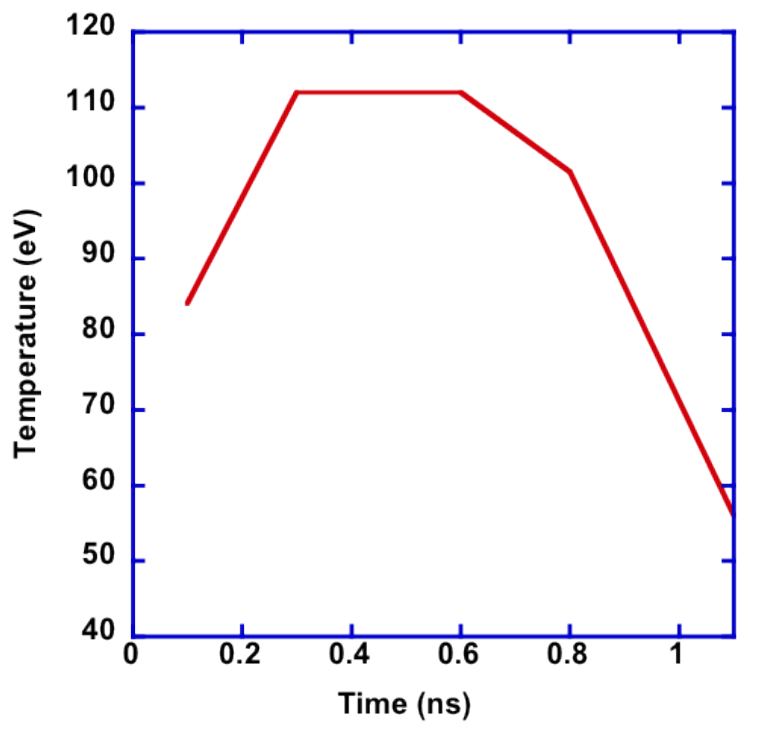

Figure 4: temperature drive

The top of figure 5 shows the density profile of the CRASH simulations results at $4 \mathrm{~ns}$ for a 1-atmosphere $\mathrm{Xe}$ gas ambient medium, a $500 \mu \mathrm{m}$ diameter, $0.25 \mathrm{~g} / \mathrm{cm}^{3}$ density $\mathrm{C}$ sphere with the temperature source from Figure 4 incident from the left. Synthetic radiographs using the Ti He-alpha line $(4.7 \mathrm{keV})$ are shown in the bottom Figure 5. The simulation clearly shows the asymmetric nature of the problem. The shock is thicker on the side where the radiation source is. The sphere reaches its maximum compression at roughly $5.5 \mathrm{~ns}$.

Currently we are performing $1 \mathrm{D}$ simulations using HYADES [10] and HELIOS-CR [11] to examine the sensitivity of the experiment to the foam density and diameter and the source flux and duration. These simulations will then guide future 2D simulations.

\section{Conclusions}

We have presented the design of an experimental platform to study the same radiation-hydrodynamics one observes when radiation from a star interacts with a cloud. The fate of the cloud is dependent on the flux of radiation incident on it and the number of photon mean free paths compared to the cloud size. We considered the parameters studied by Bertoldi [5], related them to dimensionless parameters relevant to the laboratory and showed that we can access the same regime in the laboratory that is relevant to the astrophysical system. Initial experiments will develop the platform using spheres that are optically thick to the incident radiation 

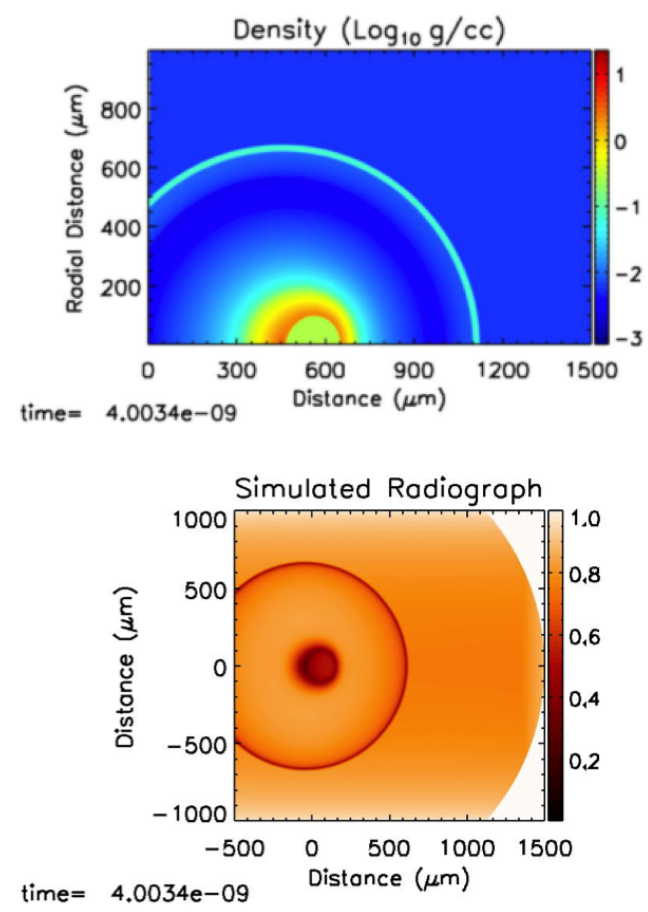

Figure 5: (Top) CRASH 2-D simulation results showing the density at 4 ns.(Bottom) Synthetic x-ray radiograph using the Ti He-alpha line $(4.7 \mathrm{keV})$ of the irradiated sphere at $4 \mathrm{~ns}$. and future experiments will reduce the optical thickness approaching the optically thin regime.

\section{Acknowledgements}

This work is funded by the U.S. Department of Energy, through the NNSA-DS and SC-OFES Joint Program in High-Energy-Density Laboratory Plasmas, grant number DE-NA0002956, and the National Laser User Facility Program, grant number DE-NA0002719, and through the Laboratory for Laser Energetics, University of Rochester by the NNSA/OICF under Cooperative Agreement No. DE-NA0001944. This work is funded by the Lawrence Livermore National Laboratory under subcontract B614207.

\section{References}

[1] J. H. Oort, L. S. Jr., The Astrophysical Journal 121 (1955) 60.

[2] F. D. Kahn, The flow of ionized gas from a globule in interstellar space, Physica 41 (1969) 172-189.

[3] R. I. Klein, M. T. S. II, R. W. Whitaker, Two-dimensional radiation-hydrodynamics calculations of the formation of $\mathrm{o}-\mathrm{b}$ associations in dense molecular clouds, Space Science Reviews 27 (1980) 275.

[4] M. T. Sandford, R. W. Whitaker, R. I. Klein, The Astrophysical Journal 260 (1982) 183-201.

[5] F. Bertoldi, Astrophys. Journ. 346 (1989) 735.

[6] O. Kessel-Deynet, A. Burket, Radiation-driven implosion of molecular cloud cores, Mon. Rot. R. Astron. Soc. 338 (2003) 545-554.

[7] T. G. Bisbas, R. Wunsch, A. P. Whitworth, D. A. Hubber, S. Walch, The Astrophysical Journal 736 (2011) 142.

[8] D. Proga, Y.-F. Jiang, S. W. Davis, J. M. Stone, , D. Smith, The Astrophysical Journal 780 (2014) 51.

[9] R. P. Drake, G. Hazak, P. A. Keiter, J. S. Davis, C. R. Patterson, A. Frank, E. G. Blackman, M. Busquet, Accepted by The Astrophysical Journal (2016).

[10] J. T. Larson, S. M. Lane, Journal of Quantitative Spectroscopy and Radiative Transfer 51 (1994) 179-186.

[11] J. J. MacFarlane, I. E. Golovkin, P. R. Woodruff, Helios-cr a 1-d radiation-magnetohydrodynamics code with inline atomic kinetics modeling, Journal of Quantitative Spectroscopy and Radiative Transfer 99 (2006) 381-397.

[12] B. van der Holst, G. Toth, I. V. Sokolov, K. G. Powell, J. P. Holloway, E. S. Myra, Q. Stout, M. L. Adams, J. E. Morel, S. Karni, B. Fryxell, R. P. Drake, The Astrophysical Journal Supplement Series. 194 (2011) 23.

[13] J. S. Davis, Y. Frank, E. Raicher, M. Fraenkel, P. A. Keiter, S. R. Klein, D. Shvarts, R. P. Drake, Review of Scientific Instruments 87 (2016). 